\title{
Front Matter: Volume 11062
}

, "Front Matter: Volume 11062," Proc. SPIE 11062, Digital Optical

Technologies 2019, 1106201 (20 September 2019); doi: 10.1117/12.2540445

SPIE. Event: SPIE Digital Optical Technologies, 2019, Munich, Germany 


\title{
Digital Optical Technologies 2019
}

\author{
Bernard C. Kress \\ Peter Schelkens \\ Editors
}

\section{4-26 June 2019 \\ Munich, Germany}

Sponsored and Published by

SPIE 
The papers in this volume were part of the technical conference cited on the cover and title page. Papers were selected and subject to review by the editors and conference program committee. Some conference presentations may not be available for publication. Additional papers and presentation recordings may be available online in the SPIE Digital Library at SPIEDigitallibrary.org.

The papers reflect the work and thoughts of the authors and are published herein as submitted. The publisher is not responsible for the validity of the information or for any outcomes resulting from reliance thereon.

Please use the following format to cite material from these proceedings:

Author(s), "Title of Paper," in Digital Optical Technologies 2019, edited by Bernard C. Kress, Peter Schelkens, Proceedings of SPIE Vol. 11062 (SPIE, Bellingham, WA, 2019) Seven-digit Article CID Number.

ISSN: 0277-786X

ISSN: 1996-756X (electronic)

ISBN: 9781510628038

ISBN: 9781510628045 (electronic)

Published by

SPIE

P.O. Box 10, Bellingham, Washington 98227-0010 USA

Telephone +13606763290 (Pacific Time) · Fax +1 3606471445

SPIE.org

Copyright @ 2019, Society of Photo-Optical Instrumentation Engineers.

Copying of material in this book for internal or personal use, or for the internal or personal use of specific clients, beyond the fair use provisions granted by the U.S. Copyright Law is authorized by SPIE subject to payment of copying fees. The Transactional Reporting Service base fee for this volume is $\$ 21.00$ per article (or portion thereof), which should be paid directly to the Copyright Clearance Center (CCC), 222 Rosewood Drive, Danvers, MA 01923. Payment may also be made electronically through CCC Online at copyright.com. Other copying for republication, resale, advertising or promotion, or any form of systematic or multiple reproduction of any material in this book is prohibited except with permission in writing from the publisher. The CCC fee code is 0277$786 \times / 19 / \$ 21.00$.

Printed in the United States of America by Curran Associates, Inc., under license from SPIE.

Publication of record for individual papers is online in the SPIE Digital Library.

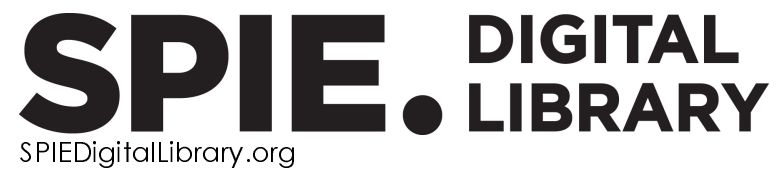

Paper Numbering: Proceedings of SPIE follow an e-First publication model. A unique citation identifier (CID) number is assigned to each article at the time of publication. Utilization of CIDs allows articles to be fully citable as soon as they are published online, and connects the same identifier to all online and print versions of the publication. SPIE uses a seven-digit CID article numbering system structured as follows:

- The first five digits correspond to the SPIE volume number.

- The last two digits indicate publication order within the volume using a Base 36 numbering system employing both numerals and letters. These two-number sets start with $00,01,02,03,04$, 05, 06, 07, 08, 09, OA, OB ... 0Z, followed by 10-1Z, 20-2Z, etc. The CID Number appears on each page of the manuscript. 


\title{
Contents
}

\author{
vii Authors \\ ix Conference Committee
}

3D DISPLAY TECHNIQUES AND TECHNOLOGIES

$1106203 \quad$ Large-scale full-color computer-generated display holograms created by stacking transferred volume holograms [1 1062-2]

1106204 Exact mask-based occlusion processing in large-scale computer holography for 3D display [1 1062-3]

1106206 Design of free-form surface backlight unit for displays [1 1062-5]

SWITCHABLE, TUNABLE AND RECONFIGURABLE OPTICS

11062 OA 1X4 VLC wavelength demultiplexer based on multislot waveguide structures [1 1062-9]

NOVEL OPTICS FOR AUGMENTED, MIXED AND VIRTUAL REALITY SYSTEMS (AR, MR, VR)

11062 OD NewSight Reality Inc. (NSR) novel transparent optical module for augmented reality eyewear (Invited Paper) [1 1062-11]

11062 OE A novel approach to freeform optimization: designing an eye-tracking augmented reality system using grid-based sag optimization [1 1062-12]

11062 OF A compact red-green-blue superluminescent diode module: A novel light source for AR microdisplays [1 1062-13]

\section{WAVEGUIDE OPTICS FOR AR/MR SYSTEMS}

$11062 \mathrm{OH} \quad$ Optical design of a thin curved lightguide and manufacturing using ophthalmic approaches (Invited Paper) [1 1062-15]

11062 0J Optical waveguide combiners for AR headsets: features and limitations [1 1062-17]

11062 OM Waveguide optics enabled consumer HUDs revolutionizing AR transportation applications [11062-72] 
DIGITAL OPTICS FOR IMAGE FORMATION

11062 ON 3D imaging systems based on projectors and mobile phones [1 1062-20]

$1106200 \quad$ PixMap: automatic license plate recognition with convolutional neural network based on saliency maps [1 1062-21]

11062 OP Holistic optimization of optical systems [1 1062-22]

$110620 Q \quad$ Research on influences of atmospheric turbulence on long-distance Fourier ptychographic imaging [1 1062-23]

INCREASING VISUAL COMFORT IN 3D DISPLAYS

11062 OT Accommodation corrected 3D displays using spatial volume demultiplexer chip [1 1062-27]

11062 OV Evaluation of augmented reality (AR) displays performance based on human visual perception [1 1062-29]

11062 oW Analysis of the visual perception conflicts in the mixed reality systems with the real-world illumination parameters restoration [1 1062-30]

DIGITAL OPTICS FOR DISPLAY AND SENSING

11062 OY Enhanced field-of-view structured illumination projector using stacked microlens arrays [11062-31]

$110620 Z$ Inspection of surface imperfections via height contrast imaging based on angle selective illumination [1 1062-32]

$1106210 \quad$ Ultra-precision angle measurement sensors with optimized size, weight and power [1 1062-33]

$1106211 \quad$ Dot pattern generation using thick sinusoidal phase grating under Gaussian beam illumination [1 1062-34]

\section{COMPUTATION DISPLAY AND IMAGING I}

$1106212 \quad$ Single exposure lensless subpixel phase imaging [1 1062-35]

$1106214 \quad$ Optical quality metrics for image restoration [1 1062-37]

iv 
1106217 A plug-n-play framework and acquisition methodology for remote exploration systems with single pixel cameras [1 1062-41]

\section{COMPUTATION DISPLAY AND IMAGING III}

11062 1D Spatially-varying blur kernel measurement based on discrete cosine transform single-pixel imaging [1 1062-47]

11062 1E Refractive telescope design with digital correction of residual chromatic aberrations [1 1062-48]

POSTER SESSION

11062 1F Design and research of lenses with a remote pupil and a telecentric beam path [1 1062-49]

$1106211 \quad$ Automatized fine-focusing in digital microscopy [1 1062-52]

$110621 \mathrm{~N} \quad$ Segmentation of illuminated areas of light using fully-convolutional neural networks and computer vision algorithms for augmented reality systems [11062-57]

1106210 Digital methods of impact on the image [1 1062-58]

11062 1P Simultaneous quantification of biomarkers using wax-patterned paper-polymer centrifugal optics [1 1062-59]

$110621 Q \quad$ Colorimetric detection of acetylcholinesterase using paper hybrid centrifugal fluidic on disc platform [1 1062-60]

$110621 \mathrm{U} \quad$ Review and analysis of optics for road lighting [1 1062-64]

11062 IV Design and aberration analysis of several AR optical architectures working with different sources of image [11062-65]

$110621 \mathrm{~A} \quad$ Achromatic image rotator [1 1062-67]

$110621 Y \quad$ The efficient method of mixed reality light restoration using HDR image of 3D scene [1 1062-68]

1106220 An extrapolation-based method for improving the accuracy of phase retrieval with the transport of intensity equation [1 1062-70] 
PLENARY SESSION

1106222 Digital optical elements and technologies (EDO19): applications to AR/VR/MR (Plenary Paper) [1 1062-76]

Proc. of SPIE Vol. 11062 1106201-6

Downloaded From: https://www.spiedigitallibrary.org/conference-proceedings-of-spie on 26 Apr 2023 Terms of Use: https://www.spiedigitallibrary.org/terms-of-use 


\section{Authors}

Numbers in the index correspond to the last two digits of the seven-digit citation identifier (CID) article numbering system used in Proceedings of SPIE. The first five digits reflect the volume number. Base 36 numbering is employed for the last two digits and indicates the order of articles within the volume. Numbers start with 00, 01, 02, 03, 04, 05, 06, 07, 08, 09, OA, OB...0Z, followed by 10-12, 20-2Z, etc.

Andreev, Lev, $1 \mathrm{~F}$

Bhattacharjee, Protim, 17

Blum, Ron, OD

Bogdanov, Nikolay N., OW, 1N, 1Y

Börner, Anko, 17

Braun, Alexander, 14

Cakmakci, Ozan, $\mathrm{OH}$

Carollo, Jerry, $\mathrm{OH}$

Castiglia, A., OF

Chukhlamov, Anton, 10

Claytor, Nelson E., 10

Dahdah, J., OF

Dannberg, Peter, OY

de Cunsel, Sébastien, OV

Derocher, Zachary, OE

Dowski, Edward Jr., 10

Duelk, M., OF

Egiazarian, Karen, 12

El Khattabi, Yassine, 00

Ezhova, Kseniia, 10

Ezhova, Vasilisa, $1 \mathrm{~F}$

Fan, Chen, 20

Fan, Xuewu, OQ

Fischer, Stephanie, OY

Fu, Qiang, 1E

Gay, Shawn, OE

Gertners, U., OT

Gindi, Moriel, OA

Gloor, S., OF

Grant, Alastair J., OM

Gupta, Amitava, OD

Hasnat, Abul, 00

Hemery, Alice, 00

Herkommer, Alois, $\mathrm{OP}$

Ikonnikov, Alexey, 1F

Jiang, Hongzhi, 1D

Johnson, Gregory, 10

Katkovnik, Vladimir, 12

Khata, Marouan, 00

Khromov, M. N., ON

Kim, Dami, 1P, 1Q

Kim, Sanghyo, 1P, 1Q

Kim, Se Jin, $1 P, 1 Q$

Knechtel, C., OZ

Kocsis, Péter, 12

Koneva, Tatiana A., $1 \mathrm{~V}$

Kress, Bernard C., OJ, 22

Kundu, Rohan, OY

Kunieda, Orie, 03
Landau, Igor, OD

Lehmann, Matthias, 14

Li, Chen, OY

Li, Chuang, $O Q$

Li, Xudong, 1D

Li, Yuxi, 1D

Llanza, Arcadi, 00

Malinverni, M., OF

Malka, Dror, OA

Martinez, Oscar A., $\mathrm{OH}$

Matsushima, Kyoji, 03, 04

Matuschek, N., OF

Meicler, Antoine, 00

Melamed, Amit, OA

Mikš, Antonín, 11

Milde, T., OZ

Moore, Ken, OE

Mounir, C., OF

Mueller, Patrick, 14

Nakamoto, K., 04

Nakib, Amir, $0 \mathrm{O}$

Narels, M., OT

Nie, Yunfeng, $1 \mathrm{E}$

Nikitin, V. G., ON

Novák, Jirí, 11

Novák, Pavel, 11

Osmanis, I., OT

Osmanis, K., OT

Ozols, A., OT

Peng, Yifan, 1E

Petrov, N. I., 06, 0N, 1X

Pokorný, Petr, 11

Popovich, Milan M., OM

Potemin, Igor S., OW, 1N, $1 \mathrm{Y}$

Primerov, N., OF

Qiao, Xuanlin, $1 \mathrm{U}$

Reichert, Carsten, OP

Rishav, Kumar, OP

Romanova, Galina E., 1F, 1U, 1V

Rossetti, M., OF

Rossi, Markus, 11

Rūtenbergs, K., OT

Samoilova, Svetlana, OD

Sanogo, Assan, 00

Scharf, Toralf, 11

Schreiber, Peter, OY

Shevkunov, Igor, 12

Shvai, Nadiya, 00

Šmejkal, Filip, 11 
Sokolov, Yu. M., ON

Sorokin, Maxim, $1 \mathrm{~N}$

Tünnermann, Andreas, OY

Vélez, C., OF

von Niederhäusern, T., OF

Waldern, Jonathan D., OM

Wang, Shuzhen, 1E

Wang, Yu, 1D

Wang, Yunfan, 1D

Xiang, Meng, $O Q$

$\mathrm{Xu}$, Yang, 1D

Yang, Mingyang, $\mathrm{OQ}$

Yousefi, Maryam, 11

Zabels, R., OT

Zeitner, Uwe, OY

Zhang, Hangying, 20

Zhang, Jingang, $1 \mathrm{E}$

Zhao, Hong, 20

Zhao, Hui, OQ

Zhao, Huijie, 1D

Zhao, Jinlei, 20

Zhao, Zixin, 20

Zhdanov, Andrey D., OW, 1N, 1Y

Zhdanov, Dmitry D., OW, 1N, 1Y

Zhuang, Yiying, 20 


\title{
Conference Committee
}

\author{
Symposium Chairs
}

Bernard C. Kress, Microsoft Corporation (United States)

Peter Schelkens, Vrije Universiteit Brussel (Belgium)

Conference Chairs

Bernard C. Kress, Microsoft Corporation (United States)

Peter Schelkens, Vrije Universiteit Brussel (Belgium)

Conference Programme Committee

Tibor Balogh, Holografika Kft. (Hungary)

Partha P. Banerjee, University of Dayton (United States)

Christian Bosshard, Centre Suisse d'Electronique et de

Microtechnique SA (Switzerland)

Arie den Boef, ASML Netherlands B.V. (Netherlands)

Federico Capasso, Harvard School of Engineering and Applied

Sciences (United States)

Oliver Cossairt, Northwestern University (United States)

Andreas Hermerschmidt, HOLOEYE Photonics AG (Germany)

Yoshio Hayasaki, Utsunomiya University (Japan)

Hans Peter Herzig, Ecole Polytechnique Fédérale de Lausanne (Switzerland)

Hong Hua, James C. Wyant College of Optical Sciences (United States)

Fu-Chung Huang, nVIDIA Corporation (United States)

Bahram Javidi, University of Connecticut (United States)

Sabina Jeschke, Rheinisch-Westfälische Technische Hochschule Aachen (Germany)

Norbert Kerwien, Carl Zeiss AG (Germany)

Joel S. Kollin, Microsoft Corporation (United States)

Byoungho Lee, Seoul National University (Korea, Republic of)

Scott McEldowney, Facebook/Oculus VR, LLC (United States)

Juan C. Minano, Limbak 4PI S.L. (Spain)

Ilmars Osmanis, Lightspace Technologies, SIA (Latvia)

Silvania F. Pereira, Technische Universiteit Delft (Netherlands)

Christophe Peroz, Magic Leap, Inc. (United States)

Pascal Picart, Université du Maine (France)

Ting-Chung Poon, Virginia Polytechnic Institute and State University (United States)

Demetri Psaltis, Ecole Polytechnique Fédérale de Lausanne

(Switzerland) 
Monika Ritsch-Marte, Medizinische Universität Innsbruck (Austria)

Robert E. Stevens, Adlens Ltd. (United Kingdom)

Hagen Stolle, SeeReal Technologies GmbH (Germany)

Adrian Travis, Microsoft Research (France)

Reinhard Voelkel, SUSS MicroOptics SA (Switzerland)

Angus Wu, Huawei Technologies Co., Ltd. (United States)

Frank Wyrowski, LightTrans International UG (Germany)

\section{Session Chairs}

1 3D Display Techniques and Technologies

Bernard C. Kress, Microsoft Corporation (United States)

2 Switchable, Tunable and Reconfigurable Optics

Svetlana Samoilova, NewSight Reality (United States)

3 Novel Optics for Augmented, Mixed and Virtual Reality Systems

(AR, MR, VR)

Christian Hellmann, Wyrowski Photonics UG (Germany)

$4 \quad$ Waveguide Optics for AR/MR Systems

Sébastien de Cunsel, WaveOptics, Ltd. (United Kingdom)

5 Digital Optics for Image Formation

Aleksandra M. Pedraszewska, VividQ (United Kingdom)

6 Increasing Visual Comfort in 3D Displays

Bernard C. Kress, Microsoft Corporation (United States)

7 Digital Optics for Display and Sensing

Thomas Milde, Carl Zeiss AG (Germany)

8 Computation Display and Imaging I

Peter Schelkens, Vrije Universiteit Brussel (Belgium)

9 Computation Display and Imaging II

Ishan Chatterjee, Microsoft Corporation (United States)

10 Computation Display and Imaging III

Bernard C. Kress, Microsoft Corporation (United States) 